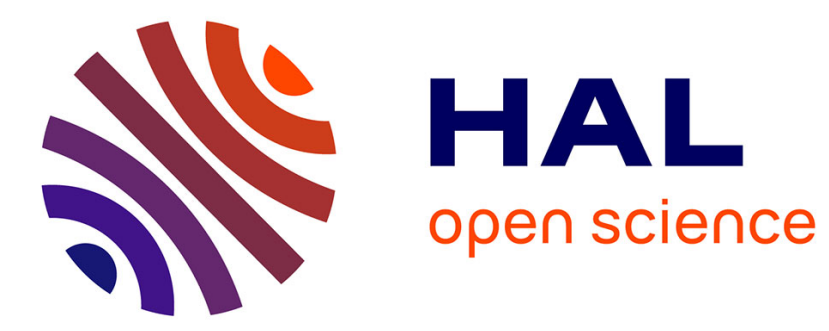

\title{
Quantum state discrimination and enhancement by noise
}

François Chapeau-Blondeau

\section{To cite this version:}

François Chapeau-Blondeau. Quantum state discrimination and enhancement by noise. Physics Letters A, 2014, 378, pp.2128-2136. 10.1016/j.physleta.2014.05.025 . hal-02171102

\section{HAL Id: hal-02171102 \\ https://hal.science/hal-02171102}

Submitted on 22 Jun 2021

HAL is a multi-disciplinary open access archive for the deposit and dissemination of scientific research documents, whether they are published or not. The documents may come from teaching and research institutions in France or abroad, or from public or private research centers
L'archive ouverte pluridisciplinaire HAL, est destinée au dépôt et à la diffusion de documents scientifiques de niveau recherche, publiés ou non, émanant des établissements d'enseignement et de recherche français ou étrangers, des laboratoires publics ou privés. 


\title{
Quantum state discrimination and enhancement by noise
}

\author{
François Chapeau-Blondeau
}

Laboratoire Angevin de Recherche en Ingénierie des Systèmes (LARIS), Université d'Angers, 62 avenue Notre Dame du Lac, 49000 Angers, France

\begin{abstract}
Discrimination between two quantum states is addressed as a quantum detection process where a measurement with two outcomes is performed and a conclusive binary decision results about the state. The performance is assessed by the overall probability of decision error. Based on the theory of quantum detection, the optimal measurement and its performance are exhibited in general conditions. An application is realized on the qubit, for which generic models of quantum noise can be investigated for their impact on state discrimination from a noisy qubit. The quantum noise acts through random application of Pauli operators on the qubit prior to its measurement. For discrimination from a noisy qubit, various situations are exhibited where reinforcement of the action of the quantum noise can be associated with enhanced performance. Such implications of the quantum noise are analyzed and interpreted in relation to stochastic resonance and enhancement by noise in information processing.
\end{abstract}

\section{Introduction}

Quantum states naturally arise when one wants to process, store or retrieve information at the level of quantum objects, such as individual photons, electrons, ions or atoms. Information processing with such quantum systems is a field of recent development, and is currently the subject of intense research, with rich potentialities [1,2]. The statistical theory of information after Shannon has been applied to quantum systems to explore some of their capabilities for information processing and communication $[3,4,1,2]$.

Another direction of recent interest at the interface between physics and information processing is the field of stochastic resonance or effects of enhancement by noise. In its early forms, stochastic resonance represents an enhancement of the response of a dynamical system occurring at an intermediate level of noise [5-7]. Stochastic resonance has progressively been shown feasible in a large variety of forms, in many systems and processes, with various measures of performance receiving enhancement by noise $[6,7]$, and new extensions are regularly reported [8-10]. As a result, in an extended sense we adopt here, stochastic resonance can be understood as a situation where enhancement of the performance in some definite task can be gained from the action of noise. For information processing, stochastic resonance as an enhancement by noise has been reported in different specific tasks, such as signal transmission [11-13], detection [14-19], estima-

E-mail address: chapeau@univ-angers.fr. tion [20,21], sensor arrays [22-25], or in relation to the statistical theory of information [26-30], although mostly in a classical context. By contrast, stochastic resonance in a quantum context has been addressed by relatively much fewer studies. Early studies on quantum stochastic resonance have considered dynamics in a double-well potential of a time-dependent position operator driven by a periodic forcing and coupled to a heat bath [31-34]. More recently, stochastic resonance has been considered in relation to binary information transmission over noisy quantum channels [35-39]. The analyses of [35-39] exhibit some possibilities of stochastic resonance or enhancement by noise in qubit communication over quantum channels assessed by mutual information, fidelity or transmission rate.

In the present study we will consider an even more basic and fundamental informational operation on quantum systems. We will analyze a discrimination process between two alternative quantum states, which can also be referred to as a quantum detection process [40-48]. In such a binary discrimination or detection process, a quantum system can be in one of two possible states; and from a measurement with two outcomes, a binary decision is taken about which quantum state the system is in $[40,41,49]$. Concerning quantum state discrimination in general, another distinct problem consists in unambiguous state discrimination [50-52,48]. Unambiguous state discrimination admits measurements that are not conclusive about which state the system is in. By contrast, the type of quantum state discrimination we investigate here, requires a decision about which state the system is in, each time a measurement is performed. In this way, quantum state detection here will designate a conclusive discrimination between two alternative 
quantum states. Imposing a conclusive discrimination exposes to detection errors, and the overall probability of detection error is taken as the performance to be optimized. Based on the theory of quantum detection $[40,41]$, the optimal measurement and its performance are exhibited in general conditions. An application is realized to optimal state discrimination on a qubit. The qubit is a fundamental quantum system of reference with important significance for quantum information. The case of the qubit, which can be worked out in detail, will allow us to test generic models of quantum noise which can affect the discrimination and its performance. The quantum noise is modeled as a noise channel acting on the qubit prior to measurement. Quantum state discrimination in this way is performed from a noisy qubit. The probability of error of the optimal detector operating on the noisy qubit will be analyzed in relation to stochastic resonance and enhancement by noise in information processing. Stochastic resonance is understood here in the broad sense of a noise-enhanced performance, much as for instance in [35-37,39] for the quantum context. Yet the present study here represents the first exploration of its kind of stochastic resonance or favorable noise effects in quantum state detection. Quantum state detection as understood here with no inconclusive measurement, matches the problem of signal detection in the sense of classical (non-quantum) statistical information processing $[15,16,49]$. Stochastic resonance or enhancement by noise has been shown feasible in classical detection problems [14-19]; and it is investigated here for the first time for quantum detection.

\section{Optimal discrimination between two quantum states}

As a standard detection situation [41,49], we assume that a quantum system, with complex Hilbert space $\mathcal{H}_{N}$ of dimension $N$, can be in one of two possible quantum states. These two quantum states can be pure states or mixed states, and are generally represented by the two (Hermitian positive unit-trace) density operators $\rho_{0}$ and $\rho_{1}$. The system can be in state $\rho_{0}$ or $\rho_{1}$ respectively with known prior probabilities $P_{0}$ or $P_{1}=1-P_{0}$, as a result of its preparation. The detection problem is to determine, from a single non-repeated measurement, whether the quantum system is in state $\rho_{0}$ or $\rho_{1}$ [41]. A generalized measurement [1] is performed on the system by means of a positive operator-valued measure (POVM) with two elements $\left\{M_{0}, M_{1}\right\}$. Each of the two POVM elements $\mathrm{M}_{k}$, for $k=0,1$, is a positive Hermitian operator satisfying $0 \leq \mathrm{M}_{k} \leq \mathbb{1}$, and together summing to the identity operator $\mathbb{1}=\mathrm{M}_{0}+\mathrm{M}_{1}$. When the measurement outcome corresponding to $\mathrm{M}_{k}$ is obtained, then it is decided that the quantum system is in state $\rho_{k}$, for $k=0,1$. The POVM contains exactly two elements because the detection problem imposes that each time a measurement is performed, a conclusive decision has to be obtained on whether the quantum system is in state $\rho_{0}$ or $\rho_{1}$. By contrast, unambiguous state discrimination as evoked in the Introduction, would generally include a third POVM element corresponding to the situation where no conclusive decision is returned on the state of the quantum system. Imposing a conclusive measurement usually exposes to detection errors. Except in the special case where the supports of $\rho_{0}$ and $\rho_{1}$ span orthogonal subspaces, the two quantum states in general cannot be perfectly distinguished, and any conclusive measurement for detection has to cope with some level of error. A relevant task is then to devise optimal strategies to minimize such errors.

The optimal detection strategies under various criteria are characterized in $[40,41,43,44]$. In this section, we exploit and adapt the results of $[40,41]$ for detection under minimum probability of detection error. Several aspects of such quantum detection have been developed in different directions, for instance in $[42,45,47,44,53$, $46,54]$. Here, we consider minimum probability-of-error detection in relation to stochastic resonance and enhancement by quantum noise, which is an original perspective. To obtain the overall probability of detection error, we have the conditional probability of each detection decision which is given by the operator trace [1]

$\operatorname{Pr}\left\{\mathrm{M}_{k} \mid \rho_{j}\right\}=\operatorname{tr}\left(\rho_{j} \mathrm{M}_{k}\right), \quad j=0,1, k=0,1$.

The overall probability of detection error $P_{\mathrm{er}}=\operatorname{Pr}\left\{\mathrm{M}_{1} \mid \rho_{0}\right\} P_{0}+$ $\operatorname{Pr}\left\{\mathrm{M}_{0} \mid \rho_{1}\right\} P_{1}$ then results as

$$
\begin{aligned}
P_{\text {er }} & =\operatorname{tr}\left(\rho_{0} \mathrm{M}_{1}\right) P_{0}+\operatorname{tr}\left(\rho_{1} \mathrm{M}_{0}\right) P_{1} \\
& =\operatorname{tr}\left[\rho_{0} \mathrm{M}_{1} P_{0}+\rho_{1}\left(\mathbb{1}-\mathrm{M}_{1}\right) P_{1}\right] \\
& =P_{1}-\operatorname{tr}\left[\left(P_{1} \rho_{1}-P_{0} \rho_{0}\right) \mathrm{M}_{1}\right]
\end{aligned}
$$

since $\mathrm{M}_{0}=\mathbb{1}-\mathrm{M}_{1}$ and the $\rho_{j}$ 's are with unit trace. From Eq. (4), the probability of detection error can also be expressed as

$P_{\mathrm{er}}=P_{1}-\operatorname{tr}\left(\mathrm{TM}_{1}\right)$,

with the test operator

$\mathrm{T}=P_{1} \rho_{1}-P_{0} \rho_{0}$,

which is Hermitian but not generally a density operator since $\mathrm{T}$ is not positive in general.

We then seek the optimal POVM $\left\{\mathrm{M}_{0}=\mathbb{1}-\mathrm{M}_{1}, \mathrm{M}_{1}\right\}$ that minimizes the probability of detection error $P_{\mathrm{er}}$ from Eq. (5). This is achieved by finding the POVM element $M_{1}$ that maximizes the term $\operatorname{tr}\left(\mathrm{TM}_{1}\right)$ in the right-hand side of Eq. (5). To characterize this optimal POVM element, the spectral decomposition of the test operator $\mathrm{T}$ is introduced as

$\mathrm{T}=\sum_{n=1}^{N} \lambda_{n}\left|\lambda_{n}\right\rangle\left\langle\lambda_{n}\right|$,

with the eigenvectors $\left\{\left|\lambda_{n}\right\rangle\right\}$ of the Hermitian operator T forming an orthonormal basis. One then gets

$\operatorname{tr}\left(\mathrm{TM}_{1}\right)=\sum_{n=1}^{N} \lambda_{n} \operatorname{tr}\left(\left|\lambda_{n}\right\rangle\left\langle\lambda_{n}\right| \mathrm{M}_{1}\right)=\sum_{n=1}^{N} \lambda_{n}\left\langle\lambda_{n}\left|\mathrm{M}_{1}\right| \lambda_{n}\right\rangle$.

Each scalar $\left\langle\lambda_{n}\left|\mathrm{M}_{1}\right| \lambda_{n}\right\rangle$ in Eq. (8) is a real confined between 0 and 1 , since $M_{1}$ is a positive operator verifying $0 \leq M_{1} \leq \mathbb{1}$. For each $n$, the maximum value of 1 is reached by $\left\langle\lambda_{n}\left|\mathrm{M}_{1}\right| \lambda_{n}\right\rangle$ when $\mathrm{M}_{1}$ is the projector $\left|\lambda_{n}\right\rangle\left\langle\lambda_{n}\right|$ on the eigensubspace spanned by $\left|\lambda_{n}\right\rangle$. The POVM element $M_{1}$ maximizing the sum in the right-hand side of Eq. (8) is thus realized by summing the rank-one projectors $\left|\lambda_{n}\right\rangle\left\langle\lambda_{n}\right|$ for all the eigenvectors $\left|\lambda_{n}\right\rangle$ associated with a positive eigenvalue $\lambda_{n}$, i.e.

$\mathrm{M}_{1}^{\mathrm{opt}}=\sum_{\lambda_{n}>0}\left|\lambda_{n}\right\rangle\left\langle\lambda_{n}\right|$

achieving for $\operatorname{tr}\left(\mathrm{TM}_{1}\right)$ in Eq. (8) the maximum $\sum_{\lambda_{n}>0} \lambda_{n}$. The optimal POVM element $\mathrm{M}_{1}^{\text {opt }}$ defined by Eq. (9) is thus the projector on the subspace spanned by the eigenvectors $\left|\lambda_{n}\right\rangle$ associated with all the positive eigenvalues $\lambda_{n}$ of the test operator T. The complementary element $\mathrm{M}_{0}^{\mathrm{opt}}=\mathbb{1}-\mathrm{M}_{1}^{\mathrm{opt}}$ is the projector on the orthogonal subspace.

The optimal POVM defined by Eq. (9) achieves in Eq. (5) the minimal probability of error

$P_{\mathrm{er}}^{\min }=P_{1}-\sum_{\lambda_{n}>0} \lambda_{n}$,

which subtracts from the prior $P_{1}$ all the positive eigenvalues of the test operator T. Since the eigenvalues $\lambda_{n}$ sum to $\operatorname{tr}(\mathrm{T})=$ $P_{1}-P_{0}$, one has equivalently $P_{\mathrm{er}}^{\mathrm{min}}=P_{0}+\sum_{\lambda_{n}<0} \lambda_{n}$ summing over 
the negative eigenvalues of $\mathrm{T}$, or by combining with Eq. (10), since $P_{0}+P_{1}=1$, [53]

$P_{\mathrm{er}}^{\min }=\frac{1}{2}\left(1-\sum_{n=1}^{N}\left|\lambda_{n}\right|\right)=\frac{1}{2}[1-\operatorname{tr}(|\mathrm{T}|)]$.

By introducing the trace distance of two operators $[1,2]$

$D\left(\rho_{0}, \rho_{1}\right)=\frac{1}{2} \operatorname{tr}\left(\left|\rho_{0}-\rho_{1}\right|\right)$,

Eq. (11) is also

$P_{\mathrm{er}}^{\min }=\frac{1}{2}-D\left(P_{0} \rho_{0}, P_{1} \rho_{1}\right)$.

This shows that the performance $P_{\mathrm{er}}^{\min }$ of the optimal detector is not solely controlled by a distance measure between the two density operators $\rho_{0}, \rho_{1}$, like the trace distance $D\left(\rho_{0}, \rho_{1}\right)$, but it also depends on the prior probabilities $\left(P_{0}, P_{1}\right)$, altogether through the trace distance $D\left(P_{0} \rho_{0}, P_{1} \rho_{1}\right)$. In a similar way, the performance $P_{\mathrm{er}}^{\mathrm{min}}$ is not completely determined by the orthogonality or nonorthogonality of the two states $\rho_{0}$ and $\rho_{1}$, as assessed for instance by the inner product $\operatorname{tr}\left(\rho_{0}^{\dagger} \rho_{1}\right)[1]$, nor is it determined by a measure of the states' purity like $\operatorname{tr}\left(\rho_{k}^{2}\right)[1]$.

From a practical standpoint, one can consider a large number of successive independent experiments, where in each experiment the quantum system is prepared either in state $\rho_{0}$ or $\rho_{1}$ with probabilities $P_{0}$ or $P_{1}$. In each experiment, from a single non-repeated measurement on the quantum system, a conclusive binary decision has to be returned on whether the preparation determined $\rho_{0}$ or $\rho_{1}$. Then, the optimal measurement strategy minimizing $P_{\mathrm{er}}$ as established in this section, guarantees the minimum number of errors over a large number of successive independent experiments; any other measurement strategy will lead to a higher number of errors.

We will now apply the general characterization of this section to the optimal discrimination of two quantum states of a qubit, where in particular the optimal performance $P_{\mathrm{er}}^{\mathrm{min}}$ of Eq. (11) can be explicitly worked out as a function of the properties of the qubit.

\section{Optimal discrimination on a qubit}

The quantum system is a qubit for which the two possible states in $\mathcal{H}_{2}$ are represented in the general Bloch sphere representation [1] as

$\rho_{j}=\frac{1}{2}\left(\mathbb{1}+\vec{r}_{j} \vec{\sigma}\right), \quad j=0,1$,

with the two real 3-dimensional Bloch vectors $\vec{r}_{j}$ of Euclidean norm $\left\|\vec{r}_{j}\right\| \leq 1$, and $\vec{\sigma}$ a vector assembling the three $2 \times 2$ (Hermitian unitary) Pauli matrices $\left[\sigma_{x}, \sigma_{y}, \sigma_{z}\right]=\vec{\sigma}$. The qubit states $\rho_{j}$ of Eq. (14) have eigenvalues $\left(1 \pm\left\|\vec{r}_{j}\right\|\right) / 2$ and determinant $\operatorname{det}\left(\rho_{j}\right)=\left(1-\left\|\vec{r}_{j}\right\|^{2}\right) / 4$.

For the detection process operating on the qubit, the test operator T of Eq. (6) follows as

$\mathrm{T}=\frac{1}{2}\left[\left(P_{1}-P_{0}\right) \mathbb{1}+\left(P_{1} \vec{r}_{1}-P_{0} \vec{r}_{0}\right) \vec{\sigma}\right]$.

The two eigenvalues of $\mathrm{T}$ then come out as

$\lambda_{ \pm}=\frac{1}{2}\left(P_{1}-P_{0} \pm\left\|P_{1} \vec{r}_{1}-P_{0} \vec{r}_{0}\right\|\right)$.

To monitor the sign of the eigenvalues of $\mathrm{T}$, we have their product

$\lambda_{+} \lambda_{-}=\operatorname{det}(\mathrm{T})=\frac{1}{4}\left[\left(P_{1}-P_{0}\right)^{2}-\left\|P_{1} \vec{r}_{1}-P_{0} \vec{r}_{0}\right\|^{2}\right]$.
It then follows that for the optimal detection operating on the qubit, there are two regimes of operation conditioning the minimal probability of detection error $P_{\mathrm{er}}^{\mathrm{min}}$ of Eqs. (10)-(11).

The first regime is when $\operatorname{det}(\mathrm{T})<0$, i.e. when $\left(P_{1}-P_{0}\right)^{2}<$ $\left\|P_{1} \vec{r}_{1}-P_{0} \vec{r}_{0}\right\|^{2}$, there is then only one positive eigenvalue $\lambda_{+}=$ $\left(P_{1}-P_{0}+\left\|P_{1} \vec{r}_{1}-P_{0} \vec{r}_{0}\right\|\right) / 2$ (the other $\lambda_{-}$is negative), and in Eq. (10) $P_{\mathrm{er}}^{\min }=P_{1}-\lambda_{+}$follows as

$P_{\mathrm{er}}^{\min }=\frac{1}{2}\left(1-\left\|P_{1} \vec{r}_{1}-P_{0} \vec{r}_{0}\right\|\right), \quad$ when $\operatorname{det}(\mathrm{T})<0$.

In this regime, in particular $P_{\mathrm{er}}^{\min }$ of Eq. (18) verifies $P_{\mathrm{er}}^{\mathrm{min}}<$ $\min \left(P_{0}, P_{1}\right)$.

The second regime is when $\operatorname{det}(\mathrm{T}) \geq 0$, i.e. when $\left(P_{1}-P_{0}\right)^{2} \geq$ $\left\|P_{1} \vec{r}_{1}-P_{0} \vec{r}_{0}\right\|^{2}$, then the two eigenvalues have the same sign. Moreover, when $P_{1}-P_{0} \geq 0$ the two eigenvalues in Eq. (16) are $\geq 0$ and sum to $\lambda_{-}+\lambda_{+}=\operatorname{tr}(\mathrm{T})=P_{1}-P_{0}$, which yields in Eq. (10) the probability of detection error $P_{\mathrm{er}}^{\min }=P_{0}=\min \left(P_{0}, P_{1}\right)$. On the contrary, when $P_{1}-P_{0} \leq 0$ the two eigenvalues in Eq. (16) are $\leq 0$ and the probability of detection error in Eq. (10) reduces to $P_{\mathrm{er}}^{\min }=P_{1}=\min \left(P_{0}, P_{1}\right)$. We thus have uniformly, in the regime when $\operatorname{det}(T) \geq 0$, the minimal probability of detection error

$P_{\mathrm{er}}^{\min }=\min \left(P_{0}, P_{1}\right), \quad$ when $\operatorname{det}(\mathrm{T}) \geq 0$.

This is the regime where the two quantum states $\rho_{0}$ and $\rho_{1}$ are sufficiently close to each other, in such a way that the measurement does not help in distinguishing them [55]. The optimal detector cannot do better than $P_{\mathrm{er}}^{\min }=\min \left(P_{0}, P_{1}\right)$ which is a probability of error that can be achieved by a prior uninformed strategy that uses no measurement but always decides for the state with maximum prior probability.

\section{Quantum noise channel}

We now consider the possibility of some noise affecting the quantum states before measurement, and we want to study the impact of such noise on the performance in discriminating the two quantum states. The action of the quantum noise on the qubit will be modeled by means of a quantum channel, which is a standard approach for quantum noise $[1,2]$. We will especially test generic models of quantum noise which can affect the qubit, represented by various models of quantum channel. Our study will encompass the cases of the bit-flip and of the depolarizing channels. In addition, we will generally consider a quantum channel realized as a convex combination of a bit-flip channel and a depolarizing channel. This produces a valid generic Pauli channel, allowing us to represent a broader class of noise processes on the qubit, in a tunable way, as also done in [39]. The combination can be tuned to represent the bit-flip or the depolarizing channels, and also a whole range of feasible Pauli channels in-between.

The action of the bit-flip channel [1,2] leaves the qubit state $\rho$ unchanged with probability $1-p$ while it applies the Pauli operator $\sigma_{x}$ flipping the quantum state with probability $p$. This can be represented by the quantum operation

$\mathcal{N}_{1}(\rho)=(1-p) \rho+p \sigma_{x} \rho \sigma_{x}^{\dagger}$.

The action of the depolarizing channel [1,2] leaves the qubit state $\rho$ unchanged with probability $1-p$ while it randomly applies any one of the three Pauli operators with probability $p / 3$. This can be represented by the quantum operation

$\mathcal{N}_{2}(\rho)=(1-p) \rho+\frac{p}{3}\left(\sigma_{x} \rho \sigma_{x}^{\dagger}+\sigma_{y} \rho \sigma_{y}^{\dagger}+\sigma_{z} \rho \sigma_{z}^{\dagger}\right)$.

Both the bit-flip channel and the depolarizing channel stand as common representations of a noise process possibly affecting 
a qubit as a result of its interaction with an uncontrolled environment. For more flexibility in the noise scenarios, we consider the possibility of a tunable convex combination of these two basic channels, realizing the quantum operation $\mathcal{N}(\rho)=(1-q) \mathcal{N}_{1}(\rho)+$ $q \mathcal{N}_{2}(\rho)$, with the mixing parameter $q \in[0,1]$. This corresponds for the noise process to the quantum operation

$$
\begin{aligned}
\mathcal{N}(\rho)= & (1-p) \rho+p\left(1-\frac{2}{3} q\right) \sigma_{x} \rho \sigma_{x}^{\dagger} \\
& +\frac{1}{3} p q\left(\sigma_{y} \rho \sigma_{y}^{\dagger}+\sigma_{z} \rho \sigma_{z}^{\dagger}\right) .
\end{aligned}
$$

At $q=0$ one recovers the bit-flip channel, while at $q=1$ one recovers the depolarizing channel. For $q$ between 0 and 1 , one has access to valid generic Pauli channels describing the effect of some external quantum noise. This same possibility of a tunable convex combination of two quantum noise channels is also used in [39], with the same motivation of examining a whole class of quantum channels by varying the parameter $q$. The parameterization by $q$ of the quantum noise channel in Eq. (22) also includes the possibility of confrontation with [37,36] where the bit-flip and depolarizing channels are addressed separately. In Eq. (22), for all $q$, the limit $p \rightarrow 0$ is equivalent to vanishing action of the noise channel with a state $\rho$ which is unaffected.

For an input qubit state $\rho$ characterized by a Bloch vector $\vec{r}=\left[r_{x}, r_{y}, r_{z}\right]^{\top}$ in the Bloch representation similar to Eq. (14), the action of the noise channel in Eq. (22) produces an output state $\mathcal{N}(\rho)$ characterized by a Bloch vector

$\vec{r}^{\prime}=\left[\alpha_{x} r_{x}, \alpha_{y} r_{y}, \alpha_{z} r_{z}\right]^{\top}$

with the scalar coefficients

$\alpha_{x}=1-\frac{4}{3} p q$,

$\alpha_{y}=\alpha_{z}=1-2 p+\frac{2}{3} p q$.

The transformed Bloch vectors resulting from Eqs. (23)-(25) can then be used in Eqs. (17)-(19) for the two qubit states, in order to assess the impact of the quantum noise on the performance in their discrimination. An interest of the approach is to explicitly materialize a model for the noise acting on the qubit prior to the detection process. The noisy qubit, after alteration by the noise channel, has its state still represented by a density operator following from Eq. (22). For this reason, the optimal detection operating on the noisy qubit is equally characterized by the analysis of Section 3. However, with the noise channel model, we have a handle to examine the effect of noise on the performance in state discrimination from a noisy qubit. This point of view is not common to the previous approaches on quantum state detection or discrimination, which usually do not explicitly incorporate a model of noise in the process. The approach here will allow us an analysis of the impact of noise, especially in relation to stochastic resonance and possibilities of enhancement by noise. In the analysis, the amount of noise will be assessed by the probability $p$ or action of the noise channel, and upon reinforcing the action of the noise channel through an increase in $p$, we will examine the evolution of the performance in the detection process.

\section{Channel action on two qubit states}

A conceivable mode of action of the noise can be described through a common communication protocol which follows. The qubit is prepared in an initial state which is either $\rho_{0}$ or $\rho_{1}$, and is sent over a noisy communication channel governed by Eq. (22). Then at the channel output, the transformed noisy qubit with state either $\mathcal{N}\left(\rho_{0}\right)$ or $\mathcal{N}\left(\rho_{1}\right)$, is measured in order to decide whether $\rho_{0}$ or $\rho_{1}$ was emitted as input. The optimal detection operates according to the analysis of Section 3 applied to the two qubit states $\mathcal{N}\left(\rho_{0}\right)$ or $\mathcal{N}\left(\rho_{1}\right)$. The two possible output Bloch vectors resulting from Eqs. (23)-(25) for $\mathcal{N}\left(\rho_{0}\right)$ or $\mathcal{N}\left(\rho_{1}\right)$ are used in Eqs. (17)-(19) to obtain the performance $P_{\mathrm{er}}^{\mathrm{min}}$ of the optimal detection realized from the channel output.

The impact of the action of the noise channel, as the channel probability $p$ is increased, is studied on the probability of detection error $P_{\mathrm{er}}^{\mathrm{min}}$ resulting from Eqs. (18)-(19). Various configurations are accessible according to the initial states $\rho_{0}$ and $\rho_{1}$, their prior probabilities $P_{0}$ and $P_{1}$, and the channel parameter $q$. For illustration, in relation to stochastic resonance and favorable noise effects, Fig. 1 presents the performance $P_{\mathrm{er}}^{\mathrm{min}}$ of the optimal detector for discrimination between the two initial pure states $\rho_{0}=|0\rangle\langle 0|$ and $\rho_{1}=|1\rangle\langle 1|$. A notable feature in Fig. 1 is the possibility of a nonmonotonic action of the noise channel probability $p$ on the performance $P_{\mathrm{er}}^{\mathrm{min}}$. At $p=0$ in Fig. 1, there is no action of the noise channel, and the detection is perfect, with a probability of error $P_{\mathrm{er}}^{\min }=0$, for two orthogonal initial states $\rho_{0}$ and $\rho_{1}$. When $p$ starts to increase above zero, the noise channel comes into action, and this leads in Fig. 1 to an increasing probability of detection error $P_{\mathrm{er}}^{\mathrm{min}}$. At $p>0$, the action of the noise channel is to transform the two initial pure states $\rho_{0}=|0\rangle\langle 0|$ and $\rho_{1}=|1\rangle\langle 1|$, via Eq. (22), into two mixed states $\mathcal{N}\left(\rho_{0}\right)$ or $\mathcal{N}\left(\rho_{1}\right)$ which are less distinguishable, therefore entailing an increased $P_{\mathrm{er}}^{\mathrm{min}}$. In this regime, $P_{\mathrm{er}}^{\mathrm{min}}$ is governed by Eq. (18), and $P_{\mathrm{er}}^{\mathrm{min}}$ degrades (increases) as the noise channel probability $p$ grows. At some $p$ sufficiently high in Fig. 1 , the two mixed states $\mathcal{N}\left(\rho_{0}\right)$ and $\mathcal{N}\left(\rho_{1}\right)$ become so mixed that on average the measurement has no possibility of distinguishing them. The performance of the optimal detector saturates at $P_{\mathrm{er}}^{\min }=\min \left(P_{0}, P_{1}\right)$, which is the performance of the prior uninformed strategy that makes no use of measurement, as governed by Eq. (19). However, further increase in the channel probability $p$ in Fig. 1, leads to a possibility of enhancement of the detection performance by reducing the probability of error $P_{\mathrm{er}}^{\mathrm{min}}$. This possibility of reducing $P_{\mathrm{er}}^{\mathrm{min}}$ is accessible for all channel configurations with $0 \leq q<1$. Yet the reduction in $P_{\mathrm{er}}^{\min }$ gets more limited as $q$ increases, as visible in Fig. 1. At $q=0$ for the bit-flip channel, the possibility of reducing $P_{\mathrm{er}}^{\min }$ at large $p>1 / 2$ can be understood, since flipping the two qubit states with sufficient certainty preserves some capacity of distinguishing between them from their flipped versions. But interestingly, the possibility of reducing $P_{\mathrm{er}}^{\mathrm{min}}$ also exists for more harmful noise channels that are not bit-flip channels. This is true for all values of $q<1$ in Fig. 1 , especially for $q>1 / 2$ when the noise channel approaches a depolarizing channel. Only at $q=1$ for the pure depolarizing channel, does the possibility of reducing $P_{\mathrm{er}}^{\min }$ by increasing $p$ disappear, for what represents the "worst-case scenario" as far as quantum noise is concerned [2].

Other configurations exist where the performance $P_{\mathrm{er}}^{\min }$ undergoes a nonmonotonic evolution, with a possibility of enhancement, as the probability $p$ of action of the noise channel increases. Fig. 2 shows such a situation, for discrimination between an initial pure state $\rho_{0}$ and an initial mixed state $\rho_{1}$. The state $\rho_{0}$ is defined by the Bloch vector $\vec{r}_{0}=$ $\left[R_{0} \sin \left(\theta_{0}\right) \cos \left(\varphi_{0}\right), R_{0} \sin \left(\theta_{0}\right) \sin \left(\varphi_{0}\right), R_{0} \cos \left(\theta_{0}\right)\right]^{\top}$ with the radius $R_{0}=1$ for a pure state, and similarly for $\rho_{1}$ with the radius $0 \leq R_{1}<1$ for a mixed state. Fig. 2 also illustrates the impact of varying the prior probabilities $\left(P_{0}, P_{1}\right)$, which preserves the possibility of nonmonotonic evolution of the performance $P_{\mathrm{er}}^{\mathrm{min}}$. Further, the analysis of Section 3 applied with the noise channel of Eq. (22) shows that nonmonotonic evolutions of $P_{\mathrm{er}}^{\min }$ with $p$ are equally feasible when the detection process operates from two initial mixed states $\rho_{0}, \rho_{1}$.

As we mentioned earlier, two quantum states $\rho_{0}$ and $\rho_{1}$ can be perfectly distinguished with no error $\left(P_{\mathrm{er}}^{\min }=0\right)$ only when 


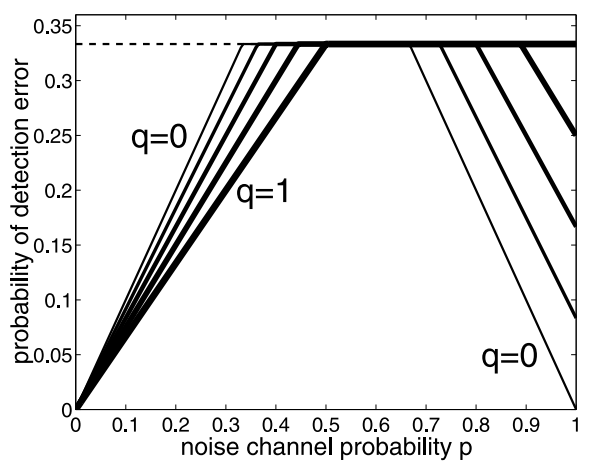

Fig. 1. For discrimination between the two initial pure states $\rho_{0}=|0\rangle\langle 0|$ and $\rho_{1}=$ $|1\rangle\langle 1|$ with prior probability $P_{1}=1 / 3$, both altered by the noise channel of Eq. (22). The solid lines are the minimal probability of error $P_{\mathrm{er}}^{\mathrm{min}}$ achieved by the optimal detector, as a function of the noise channel probability $p$ in abscissa, for five different values of the channel parameter $q=0,0.25,0.5,0.75$ and 1 with increasing thickness of the solid lines. The dashed horizontal line is $\min \left(P_{0}, P_{1}\right)=P_{1}=1 / 3$ the probability or error of the prior uninformed detection strategy.

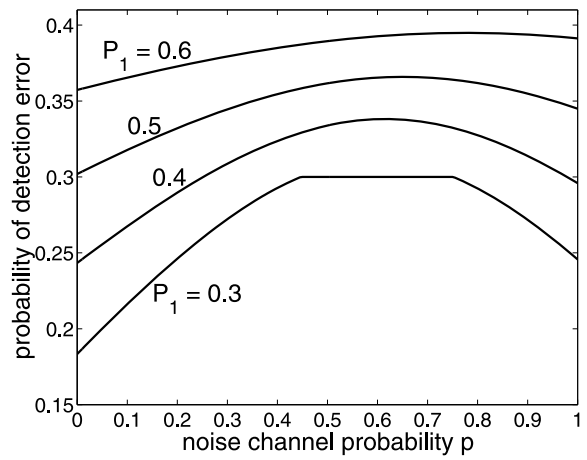

Fig. 2. For discrimination between the initial pure state $\rho_{0}$ characterized by the Bloch vector $\vec{r}_{0}=\left(R_{0}=1, \theta_{0}=0.22 \pi, \varphi_{0}=0\right)$ in spherical coordinates, and the initial mixed state $\rho_{1}$ with Bloch vector $\vec{r}_{1}=\left(R_{1}=0.3, \theta_{1}=0, \varphi_{1}=0\right)$, both altered by the noise channel of Eq. (22) with parameter $q=0.2$. The solid lines are the minimal probability of error $P_{\mathrm{er}}^{\mathrm{min}}$ achieved by the optimal detector, as a function of the noise channel probability $p$ in abscissa, for four different values of the prior probability $P_{1}=0.3,0.4,0.5$ and 0.6 .

they are orthogonal, or equivalently when their inner product $\operatorname{tr}\left(\rho_{0}^{\dagger} \rho_{1}\right)=0$. For two qubit states in $\mathcal{H}_{2}$, the inner product is $\operatorname{tr}\left(\rho_{0}^{\dagger} \rho_{1}\right)=\left(1+\vec{r}_{0} \vec{r}_{1}\right) / 2$ and orthogonality can occur only when $\vec{r}_{0}=-\vec{r}_{1}$ while $\left\|\vec{r}_{0}\right\|=\left\|\vec{r}_{1}\right\|=1$, i.e. for two pure states opposite on the Bloch sphere. This is the case for the two initial pure states $\rho_{0}=|0\rangle\langle 0|$ and $\rho_{1}=|1\rangle\langle 1|$ in Fig. 1 , which can be distinguished with no error at $P_{\mathrm{er}}^{\min }=0$, yet only when no noise channel is acting (i.e. at $p=0$ in Fig. 1). At $p>0$ the noise channel affects the two qubit states $\rho_{0}$ and $\rho_{1}$ which cease to be orthogonal; and nonorthogonality generally leads to a nonzero probability of detection error $P_{\mathrm{er}}^{\mathrm{min}}>0$ in Fig. 1. However, a measure of nonorthogonality of the two qubit states $\rho_{0}$ and $\rho_{1}$, as provided by their inner product $\operatorname{tr}\left(\rho_{0}^{\dagger} \rho_{1}\right)$, is in general not sufficient to determine the behavior of the probability of error $P_{\mathrm{er}}^{\min }$ in the detection in the presence of noise. This is illustrated in Fig. 3 which considers three distinct nonorthogonal initial configurations of the two qubit states $\rho_{0}$ and $\rho_{1}$, yet sharing all three the same inner product $\operatorname{tr}\left(\rho_{0}^{\dagger} \rho_{1}\right)=1 / 4$. The nonmonotonic evolution of the probability of error $P_{\mathrm{er}}^{\min }$ is preserved in Fig. 3 as the probability $p$ of action of the noise channel increases. Nevertheless, the three initial configurations with same inner product evolve differently in their response to the noise as assessed by $P_{\mathrm{er}}^{\mathrm{min}}$. This was anticipated from the expression of Eq. (13), showing that not only the (nonorthogonal) configurations of $\rho_{0}$ and $\rho_{1}$, but also their prior probabilities

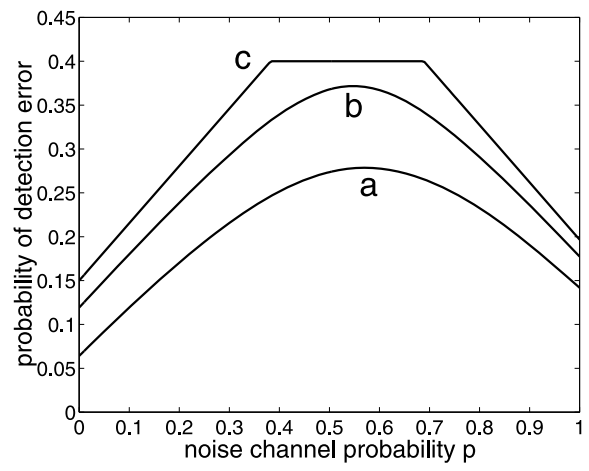

Fig. 3. For discrimination from three pairs of initial nonorthogonal qubit states $\rho_{0}$ and $\rho_{1}$ sharing the same inner product $\operatorname{tr}\left(\rho_{0}^{\dagger} \rho_{1}\right)=1 / 4$, the minimal probability of error $P_{\mathrm{er}}^{\mathrm{min}}$ achieved by the optimal detector as a function of the noise channel probability $p$ in abscissa, with prior probability $P_{1}=0.6$ and channel parameter $q=0.2$. In all three pairs $\rho_{0}=|0\rangle\langle 0|$ with Bloch vector $\vec{r}_{0}=\left(R_{0}=1, \theta_{0}=0, \varphi_{0}=0\right)$ in spherical coordinates, while $\rho_{1}$ has Bloch vector $\vec{r}_{1}$ with spherical coordinates: (a) $\left(R_{1}=1, \theta_{1}=2 \pi / 3, \varphi_{1}=0\right)$, (b) $\left(R_{1}=1 / \sqrt{2}, \theta_{1}=3 \pi / 4, \varphi_{1}=0\right)$, (c) $\left(R_{1}=\right.$ $\left.1 / 2, \theta_{1}=\pi, \varphi_{1}=0\right)$.

$P_{0}$ and $P_{1}$, have a separate impact on $P_{\mathrm{er}}^{\min }$. Yet, the interesting property of a nonmonotonic evolution of $P_{\mathrm{er}}^{\mathrm{min}}$ as $p$ increases is preserved as a common feature in Fig. 3.

A trace-preserving quantum operation as in Eq. (22) is contractive for the trace distance of Eq. (12) between two density operators [1], yielding $D\left(\mathcal{N}\left(\rho_{0}\right), \mathcal{N}\left(\rho_{1}\right)\right) \leq D\left(\rho_{0}, \rho_{1}\right)$. This holds also for the weighted operators $P_{0} \rho_{0}$ and $P_{1} \rho_{1}$ occurring in Eq. (13) [2], yielding $D\left(\mathcal{N}\left(P_{0} \rho_{0}\right), \mathcal{N}\left(P_{1} \rho_{1}\right)\right)=D\left(P_{0} \mathcal{N}\left(\rho_{0}\right), P_{1} \mathcal{N}\left(\rho_{1}\right)\right) \leq$ $D\left(P_{0} \rho_{0}, P_{1} \rho_{1}\right)$. The trace distance between the two weighted density operators therefore cannot increase by the action of the noise channel of Eq. (22). As a consequence, the probability of error $P_{\mathrm{er}}^{\mathrm{min}}$ of Eq. (13) cannot decrease by the action of the noise channel of Eq. (22). This means that the performance $P_{\mathrm{er}}^{\min }$ after the action of the noise channel of Eq. (22) cannot be better than the performance $P_{\mathrm{er}}^{\mathrm{min}}$ before the action of the channel. In other words, the optimal discrimination from the initial states $\rho_{0}$ and $\rho_{1}$ is never surpassed in performance by the optimal discrimination from the noisy output states $\mathcal{N}\left(\rho_{0}\right)$ and $\mathcal{N}\left(\rho_{1}\right)$ after action of the noise channel. This can be verified in Figs. 1-3 where the probability of error $P_{\mathrm{er}}^{\mathrm{min}}$ at $p=0$ for optimal discrimination from the initial states, is never above $P_{\mathrm{er}}^{\min }$ at any $p>0$ when the noise channel is acting to alter the two states. In this respect, if the initial qubit used for signaling is directly accessible to measurement, then there is no benefit in purposefully applying a noise channel like Eq. (22) with nonzero $p$ before measuring the qubit for detection.

However, for different levels of $p>0$ characterizing the action of the noise channel, larger values of $p$ can lead to better detection performance $P_{\mathrm{er}}^{\mathrm{min}}$ as revealed in Figs. 1-2. In this respect, if the initial qubit used for signaling is not directly accessible to measurement, but is necessarily measured after the action of a noise channel, then it may be beneficial to reinforce the action of the noise channel through purposeful increase of its probability of action $p$. This may lead, as visible in Figs. 1-3, to a better performance $P_{\mathrm{er}}^{\mathrm{min}}$ in the detection process. In brief, if no quantum noise channel preexists, then there is no benefit for the detection in adding some; however, if some quantum noise channel preexists, then it is sometimes beneficial to reinforce its action on the qubit.

\section{Channel action on one qubit state}

As another conceivable mode of action of the noise, we consider the following protocol where the action of a noise channel similar to Eq. (22) can be deliberately implemented by the sender as part of her/his preparation of the signaling states $\rho_{0}$ or $\rho_{1}$, 
prior to a noise-free communication of the qubit to the receiver. In this protocol, the noise channel is seen as a device accessible to the sender for her/his preparation of the signaling states $\rho_{0}$ or $\rho_{1}$ of the qubit. After the preparation by the sender is complete, the qubit is communicated to the receiver with no further transformation. The receiver then measures the qubit to return a binary decision. With this protocol, we want to study the possibility of some beneficial scenario when using a noise channel similar to Eq. (22) as an auxiliary device accessible to the sender for the preparation of the qubit in one or the other signaling states. This is another configuration to investigate the action of the quantum noise, and which complements the more common situation of Section 5 where the noise channel acts equally on the two states $\rho_{0}$ and $\rho_{1}$.

The sender can thus choose to transform one or the other of the signaling states $\rho_{0}$ or $\rho_{1}$ by means of a noise channel similar to Eq. (22). If the two states are transformed by the same noise channel, we are back to the situation addressed in Section 5. We will therefore consider the possibility of transforming only one of the two signaling states, prior to (perfect) communication to the receiver for detection. The Bloch vector of the unaffected state, and that of the transformed state resulting from Eqs. (23)-(25), are used in Eqs. (17)-(19) to obtain the performance $P_{\mathrm{er}}^{\mathrm{min}}$ in the optimal detection.

With this approach, Fig. 4 addresses the discrimination between the pure state $\rho_{0}=|0\rangle\langle 0|$ and an initial mixed state $\rho_{1}$. In Fig. 4 , the noise channel used in the preparation procedure to further transform the initial state $\rho_{1}$ is the depolarizing channel of Eq. (21). The depolarizing noise channel $\mathcal{N}_{2}(\cdot)$ of Eq. (21) is equivalent to Eq. (22) at $q=1$ and represents in some sense the "worst-case" noise condition as reflected in Fig. 1 . The state $\rho_{0}$ remains unchanged, while the initial state $\rho_{1}$ is progressively affected by the depolarizing noise channel of Eq. (21) prior to detection. An interesting behavior in Fig. 4 is the possibility of reducing the probability of detection error $P_{\mathrm{er}}^{\min }$ by means of an increase in the probability $p$ of action of the depolarizing noise channel of Eq. (21). In Fig. 4, at small p, the performance in detection is poor, with a probability of error $P_{\mathrm{er}}^{\mathrm{min}}$ of the optimal detector which can do no better than $\min \left(P_{0}, P_{1}\right)$ which is the probability or error of the prior uninformed detection strategy using no measurement. This can be explained by a pure state $\rho_{0}$ and a mixed state $\rho_{1}$ which are initially very close. As a result, the optimal detector operates in the regime of Eq. (19) where the measurement is of no use to enhance the performance above that of the prior uninformed strategy. Then, the increase of the probability $p$ of the depolarizing channel results in a transformed state $\mathcal{N}_{2}\left(\rho_{1}\right)$ which becomes more distinguishable from the other state $\rho_{0}$. The noise channel acts to separate the two states and improves their discrimination. As $\rho_{1}$ is progressively depolarized by increasing $p$ in Fig. 4, the probability of detection error $P_{\mathrm{er}}^{\mathrm{min}}$ diminishes. This is shown in Fig. 4 for three configurations of the initial mixed state $\rho_{1}$, illustrating the same effect of enhanced performance upon depolarization of $\rho_{1}$ by the noise channel.

A favorable action of the noise channel can also be registered when the quantum system is initially, at $p=0$, in a regime of useful measurement as in Eq. (18). Examples are shown in Fig. 5, for discrimination between the pure state $\rho_{0}=|0\rangle\langle 0|$, and a mixed state $\rho_{1}$ in different initial configurations which is progressively affected by the depolarizing noise channel $\mathcal{N}_{2}(\cdot)$ of Eq. (21). With no depolarizing noise, i.e. at $p=0$ in Fig. 5 , the probability of error $P_{\mathrm{er}}^{\mathrm{min}}$ of the optimal detector starts at a value strictly below $\min \left(P_{0}, P_{1}\right)$ the probability or error of the prior uninformed detection strategy. Then, as the depolarizing noise increases with $p$ rising above zero, the probability of error $P_{\mathrm{er}}^{\min }$ can regularly decrease as in Fig. 5(a), or it can start to temporarily increase as in Fig. 5(b)-(c). For sufficiently large $p$, the probability of error $P_{\mathrm{er}}^{\min }$

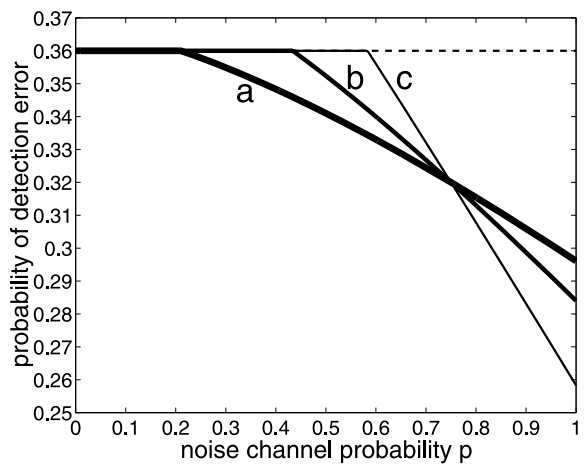

Fig. 4. For discrimination between the pure state $\rho_{0}=|0\rangle\langle 0|$ and the mixed state $\rho_{1}$ with prior probability $P_{1}=0.64$. The dashed horizontal line is $\min \left(P_{0}, P_{1}\right)=P_{0}=$ 0.36 the probability of error of the prior uninformed detection strategy. The three solid lines are the minimal probability of error $P_{\mathrm{er}}^{\min }$ achieved by the optimal detector, as a function of the noise channel probability $p$. The mixed state $\rho_{1}$ defined by the Bloch vector $\vec{r}_{1}$ is progressively affected by the depolarizing noise channel of Eq. (21) equivalent to Eq. (22) at $q=1$. Three initial configurations at $p=0$ are tested for $\vec{r}_{1}$ with spherical coordinates $\left(R_{1}, \theta_{1}, \varphi_{1}=0\right)$ : (a) $\left(R_{1}=0.3, \theta_{1}=0.25 \pi\right)$, (b) $\left(R_{1}=0.4, \theta_{1}=0.2 \pi\right)$, (c) $\left(R_{1}=0.6, \theta_{1}=0.1 \pi\right)$.

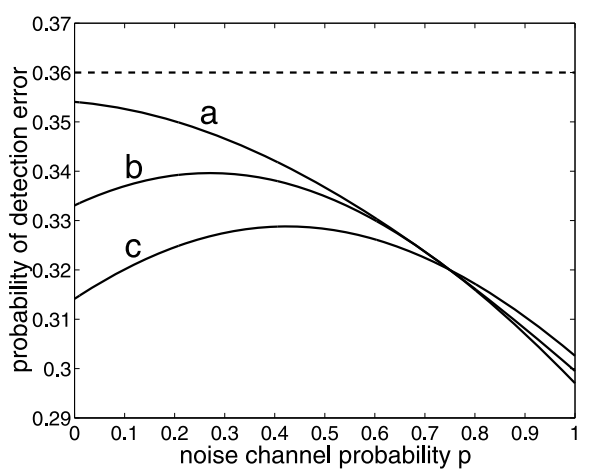

Fig. 5. Same as Fig. 4, with for $\vec{r}_{1}$ the three initial configurations, at $p=0$ : (a) $\left(R_{1}=0.3, \theta_{1}=0.3 \pi\right)$, (b) $\left(R_{1}=0.4, \theta_{1}=0.35 \pi\right)$, (c) $\left(R_{1}=0.4, \theta_{1}=0.4 \pi\right)$.

decreases as $p$ grows further, for the three configurations in Fig. 5, manifesting enhanced performance in detection by the action of the noise channel.

It can also be noted in both Fig. 4 and Fig. 5, that a nonzero depolarizing probability $p$ can enhance the detection performance strictly above its level at $p=0$ with no noise channel. Moreover, $P_{\mathrm{er}}^{\mathrm{min}}$ can achieve its overall best (smallest) value when the probability of action of the depolarizing noise channel of Eq. (21) is at its maximum $p=1$. As we argued at the end of Section 5, this outcome is not feasible when the two states are affected by the noise channel: the best performance is always with no noise channel. By contrast, when only one state is affected by the noise channel, the best performance can occur through effective action of the noise channel, which contributes to increase the distinguishability of the two states and reduces the probability of error $P_{\mathrm{er}}^{\min }$ in their discrimination.

As a third example with the protocol of this section where the noise channel acts only on the state $\rho_{1}$ as part of the preparation by the sender, we consider discrimination with a state $\rho_{0}$ which is the maximally mixed qubit state $\rho_{0}=\mathbb{1} / 2$. For discrimination between an initially pure state $\rho_{1}$ and $\rho_{0}=\mathbb{1} / 2$, Fig. 6 shows the probability of error $P_{\mathrm{er}}^{\mathrm{min}}$, as a function of the noise channel probability $p$, with various types of the noise channel at different $q$ in Eq. (22). Nonmonotonic evolutions are observed for $P_{\mathrm{er}}^{\mathrm{min}}$ in Fig. 6, at all values of $q$ fixing the type of the noise channel, with a range where an increase of the probability $p$ of action of the noise channel reduces the probability of error $P_{\mathrm{er}}^{\mathrm{min}}$. Similar nonmonotonic evolutions of the performance $P_{\mathrm{er}}^{\mathrm{min}}$ for all $q$ are preserved for dis- 


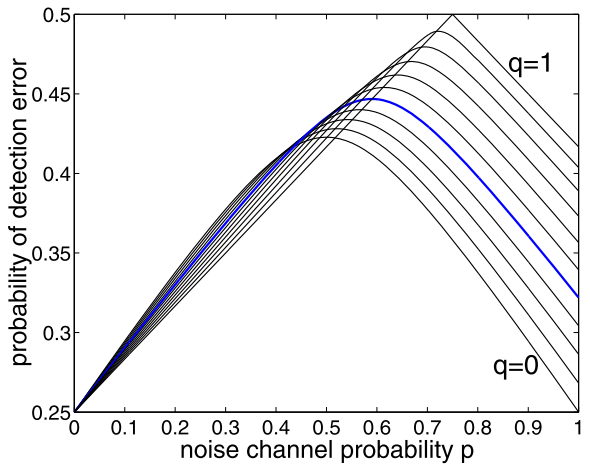

Fig. 6. For discrimination between the maximally mixed qubit state $\rho_{0}=\mathbb{1} / 2$ and the initial pure state $\rho_{1}$ with Bloch vector $\vec{r}_{1}=\left(R_{1}=1, \theta_{1}=0.1 \pi, \varphi_{1}=0\right)$ and prior probability $P_{1}=1 / 2$, the minimal probability of error $P_{\mathrm{er}}^{\min }$ achieved by the optimal detector, as a function of the noise channel probability $p$, for different values of the channel parameter $q$ from $q=0$ to $q=1$ by step 0.1 . The ticker blue line is at $q=0.4$. The noise channel of Eq. (22) only affects the state $\rho_{1}$, while the state $\rho_{0}=\mathbb{1} / 2$ is unaffected since and stands as a fixed point of the noise channel for any $p$ and $q$. (For interpretation of the references to color in this figure legend, the reader is referred to the web version of this article.)

crimination from a mixed state $\rho_{1}$, and at other values of the prior probability $P_{1} \neq 1 / 2$.

A notable feature with the signaling state $\rho_{0}=\mathbb{1} / 2$ in Fig. 6 is that this state remains invariant by the transformation of Eq. (22), i.e. $\mathcal{N}(\mathbb{1} / 2)=\mathbb{1} / 2$ for any $p$ and $q$. This is easily verified from Eq. (23) since the state $\rho_{0}=\mathbb{1} / 2$ has the invariant Bloch vector $\vec{r}_{0}=\overrightarrow{0}$. Therefore, any noise channel according to Eq. (22) has no effect on the signaling state $\rho_{0}=\mathbb{1} / 2$. In this case, the protocol of this Section 6 where the noise channel acts only on the state $\rho_{1}$, is equivalent to the more common protocol of Section 5 where the noise channel acts equally on the two states $\rho_{1}$ and $\rho_{0}=\mathbb{1} / 2$. Therefore, the nonmonotonic evolutions of the performance $P_{\mathrm{er}}^{\mathrm{min}}$ with the noise channel probability $p$ in Fig. 6, characterize the discrimination between states $\rho_{1}$ and $\rho_{0}=\mathbb{1} / 2$ after action of the noise channel, equally in the protocols of Section 5 and Section 6 . As a consequence, the argument at the end of Section 5 applies to the nonmonotonic evolutions of $P_{\mathrm{er}}^{\mathrm{min}}$ in Fig. 6 for discrimination with $\rho_{0}=\mathbb{1} / 2$ : the performance at $p>0$ (although nonmonotonic) can never surpass the performance at $p=0$, as verified in Fig. 6. For discrimination from $\rho_{0}=\mathbb{1} / 2$, reinforcing the noise by increasing $p$ can be beneficial when some quantum noise preexists, within the protocol of Section 5.

\section{Discussion}

In this report we have studied a quantum detection process as a conclusive discrimination between two alternative quantum states, in relation to stochastic resonance and effects of enhancement by noise in information processing. The optimal quantum detector minimizing the probability of detection error is exhibited in general conditions. An application is made to discrimination on the qubit, which is a fundamental reference system of quantum information theory. The case of the qubit, which can be worked out in detail, allowed us to test generic models of quantum noise, materialized by a noise channel randomly applying Pauli operators on the qubit prior to detection. In this way, the detection process was performed on a noisy qubit, and we specifically analyzed the impact of the noise on the detection performance. This specific perspective, to explicitly introduce a model for quantum noise and to focus on its impact in detection, is not common to the previous literature on quantum state detection or discrimination. This led us to identify situations which can be interpreted as possibilities of stochastic resonance or enhancement by noise in quantum state discrimination. This was manifested by conditions where an increase in the probability $p$ of action of the noise channel to affect the qubit could be associated with a reduced probability of detection error. Such a beneficial outcome can be obtained for two modes of action of the quantum noise, that we successively considered in Sections 5 and 6.

The noise channel can act on the two qubit states, as in Section 5. This represents the case of a qubit which is transmitted from the sender, through the noisy quantum channel, to the receiver performing the detection. In this mode, an increase in the probability $p$ of action of the noise channel, can lead to enhanced detection for all the noise channels tested $(0 \leq q<1)$, except for the pure depolarizing channel $(q=1)$ which represents the worse noise condition. In this mode of action of the quantum noise, it is nevertheless always preferable for detection to measure the qubit at zero noise $(p=0)$, if this is feasible; but if some inevitable nonzero noise preexists $(p>0)$, deliberately increasing to larger $p$ can sometimes be profitable to detection.

Alternatively, the noise channel can act on only one of the two quantum states, as in Section 6. This represents a case where the sender, while preparing the qubit, has a separate control on the two signaling states. In the preparation, the sender can choose to modify the qubit only when it is in one of the two signaling states. To modify the qubit, the sender deliberately applies the noise channel. After this preparation by the sender, the qubit is directly communicated with no further transformation to the receiver for detection. In this mode, an increase in the probability $p$ of action of the noise channel, can lead to enhancement in detection for all the noise channels, especially the depolarizing channel. And configurations with nonzero noise $(p>0)$ can be more efficient for detection than the absence of quantum noise $(p=0)$.

These two modes of action of the quantum noise that we have examined can naturally be combined, with two successive noise channels, one at the qubit preparation and one at the qubit transmission. Detection after the resulting process is still governed by the analysis of Section 3, and comparable effects of enhanced performance can be expected to persist.

A common explanation for the favorable action of the noise channel on the detection, can be derived from the performance $P_{\mathrm{er}}^{\mathrm{min}}$ under its form of Eq. (13). Our analysis can be viewed as revealing the impact of the probability $p$ of action of the noise channel, on the trace distance $D\left(P_{0} \rho_{0}, P_{1} \rho_{1}\right)$ between the two weighted states $P_{0} \rho_{0}, P_{1} \rho_{1}$ occurring in Eq. (13). This trace distance $D\left(P_{0} \rho_{0}, P_{1} \rho_{1}\right)$ does not share all the properties of a trace distance between two density operators, because the weighted states $P_{0} \rho_{0}, P_{1} \rho_{1}$, strictly, are not density operators; and $D\left(P_{0} \rho_{0}, P_{1} \rho_{1}\right)$ also conveys the importance of the priors $P_{0}, P_{1}$ in the detection performance. An important observation here is that the action of $p$ on $D\left(P_{0} \rho_{0}, P_{1} \rho_{1}\right)$ is nonmonotonic. An increase in the probability $p$ can sometimes, in conditions determined by the analysis of Section 3 , increase the distance $D\left(P_{0} \rho_{0}, P_{1} \rho_{1}\right)$, inducing in this way an enhancement of the detection performance $P_{\mathrm{er}}^{\min }$.

Discrimination of quantum states is a fundamental operation for information processing. This operation is studied here for the first time in relation to stochastic resonance and possibilities of enhancement by noise in a quantum context. There can be several meaningful ways of quantifying the amount of quantum noise. In the quantum context considered here, the noise essentially models the interaction of the qubit with an uncontrolled environment $[1,2]$. The probability $p$ as defined in the noise channel models of Section 4, provides a parameter which directly quantifies the amount of action of the noise channel on the qubit. At $p \rightarrow 0$, the action of the noise channel vanishes; as $p$ rises above zero, there is more and more probability that the noise channel act to alter the qubit, or equivalently the qubit is more and more exposed to alteration by the environment. This assigns a direct interpretation to 
the noise channel parameter $p$, as quantifying the probability of alteration of the qubit by the environment. Our analysis shows that there is no monotonic detrimental action of the environment for discrimination issues, and larger coupling to the environment can sometimes be found beneficial for discrimination. For instance, in some situations, a very noisy quantum state resulting from strong decoherence produced by the environment, and corresponding to large values of $p$, can lead to higher efficacy in distinguishing from another given quantum state.

The quantum systems and noise models considered in this study represent basic elements from quantum information processing and quantum information theory, as reviewed for instance in $[1,2]$. They allowed us here to show some possibilities of stochastic resonance or noise-enhanced performance in a quantum detection task operating at the level of these basic elements. These are the same basic elements of quantum information that are also used in many other studies for instance on quantum state discrimination, detection, or noise in communication with quantum channels, as referred to in this report. Also quantum measurement here has the special form of a process of decision between two alternative density operators. At another level concerning quantum noise and quantum measurement, another line of study starts from macroscopic or mesoscopic systems, like electrical circuits or resonant cavities, and describes how the quantum-limited regime can be reached and handled, as for instance addressed in [56-59]. This complementary level of approach, in connection with the present results, could be useful to identify the conditions under which the standard noise channel models of [1,2] used here arise, or to describe the implementation with physical devices of the binary measurement defined by the optimal detector of Section 2. This represents an important step to connect quantum effects identified at the elementary levels of the basic operators of quantum information [1,2], to actual physical implementation systems existing at the macroscopic level [56-59]. Mastering such connection is a very vast program, which is currently under active development, and is clearly beyond the scope of the present study. In this context of quantum information $[1,2]$ it is often the case that for the informational task at hand, the post-measurement state is not relevant, and only the result of a single non-repeated measurement matters. This is the case here for the detection task, where a binary quantum measurement is used to decide between two alternative density operators; once a decision is obtained, the postmeasurement state and the fate of the quantum system are not relevant. For this reason, the effect of measurement back-action, as for instance considered in [58], is not included in the analysis of the quantum detection task.

\section{Conclusion}

Based on the theory of quantum detection, we have described a setting for quantum state discrimination through a conclusive binary measurement operating on a noisy qubit. The noise is realized by a quantum noise channel of various types acting on the qubit prior to its measurement. The amount of noise is quantified by the probability $p$ of action of the noise channel on the qubit, and we focused on the impact of noise on the discrimination performance assessed by the probability of error of the optimal detector. We have shown the possibility of various situations where reinforcement of the action of the noise through an increase of the probability $p$ can be associated with enhanced performance in discrimination. Such outcome is interpreted as a novel instance of stochastic resonance or enhancement by noise in information processing, established here for quantum state discrimination with quantum noise. This constitutes a new step in the explorations of stochastic resonance and favorable noise effects, and their various forms and conditions.
This first study on quantum detection and stochastic resonance can be extended in several directions. Beyond the case of the qubit, and based on the general theory of Section 2, quantum systems of larger dimension can be considered, with their appropriate generalization of the Pauli noise channels [2]. Discrimination from such noisy quantum states can then be investigated for the impact of noise, and for possibilities of enhancement by quantum noise. Other quantum information processes and channels have been recently investigated for extension of stochastic resonance [60-63]. The present results contribute in the same endeavor of exploring possible benefits from noise in relation to quantum information processing.

\section{References}

[1] M.A. Nielsen, I.L. Chuang, Quantum Computation and Quantum Information, Cambridge University Press, Cambridge, 2000.

[2] M.M. Wilde, Quantum Information Theory, Cambridge University Press, Cambridge, 2013.

[3] B. Schumacher, M.A. Nielsen, Quantum data processing and error correction, Phys. Rev. A 54 (1996) 2629-2635.

[4] A.S. Holevo, The capacity of the quantum channel with general signal states, IEEE Trans. Inf. Theory 44 (1998) 269-273.

[5] M.I. Dykman, H. Haken, G. Hu, D.G. Luchinsky, R. Mannella, P.V.E. McClintock, C.Z. Ning, N.D. Stein, N.G. Stocks, Linear response theory in stochastic resonance, Phys. Lett. A 180 (1993) 332-336.

[6] L. Gammaitoni, P. Hänggi, P. Jung, F. Marchesoni, Stochastic resonance, Rev. Mod. Phys. 70 (1998) 223-287.

[7] M.D. McDonnell, N.G. Stocks, C.E.M. Pearce, D. Abbott, Stochastic Resonance: From Suprathreshold Stochastic Resonance to Stochastic Signal Quantization, Cambridge University Press, Cambridge, 2008.

[8] G. Balázsi, L.B. Kish, From stochastic resonance to brain waves, Phys. Lett. A 265 (2000) 304-316.

[9] J.P. Zbilut, T. Scheibel, D. Huemmerich, C.L. Webber Jr., M. Colafranceschi, A. Giuliani, Spatial stochastic resonance in protein hydrophobicity, Phys. Lett. A 346 (2005) 33-41.

[10] R. Storni, H. Ando, K. Aihara, K. Murali, S. Sinha, Manipulating potential wells in Logical Stochastic Resonance to obtain XOR logic, Phys. Lett. A 376 (2012) 930-937.

[11] F. Chapeau-Blondeau, Input-output gains for signal in noise in stochastic resonance, Phys. Lett. A 232 (1997) 41-48.

[12] Y. Zhang, G. Hu, L. Gammaitoni, Signal transmission in one-way coupled bistable systems: noise effect, Phys. Rev. E 58 (1998) 2952-2956.

[13] J.C. Comte, S. Morfu, Stochastic resonance: another way to retrieve subthreshold digital data, Phys. Lett. A 309 (2003) 39-43.

[14] M.E. Inchiosa, A.R. Bulsara, Signal detection statistics of stochastic resonators, Phys. Rev. E 53 (1996) R2021-R2024.

[15] D. Rousseau, F. Chapeau-Blondeau, Stochastic resonance and improvement by noise in optimal detection strategies, Digit. Signal Process. 15 (2005) 19-32.

[16] H. Chen, P.K. Varshney, S.M. Kay, J.H. Michels, Theory of stochastic resonance effect in signal detection: Part I - Fixed detectors, IEEE Trans. Signal Process. 55 (2007) 3172-3184

[17] A. Patel, B. Kosko, Optimal noise benefits in Neyman-Pearson and inequalityconstrained statistical signal detection, IEEE Trans. Signal Process. 57 (2009) $1655-1669$

[18] F. Duan, F. Chapeau-Blondeau, D. Abbott, Fisher-information condition for enhanced signal detection via stochastic resonance, Phys. Rev. E 84 (2011) 051107

[19] S. Bayram, S. Gezici, Stochastic resonance in binary composite hypothesistesting problems in the Neyman-Pearson framework, Digit. Signal Process. 22 (2012) 391-406

[20] F. Chapeau-Blondeau, Noise-aided nonlinear Bayesian estimation, Phys. Rev. E 66 (2002) 032101

[21] H. Chen, P.K. Varshney, J.H. Michels, Noise enhanced parameter estimation, IEEE Trans. Signal Process. 56 (2008) 5074-5081.

[22] N.G. Stocks, Information transmission in parallel threshold arrays: suprathreshold stochastic resonance, Phys. Rev. E 63 (2001) 041114

[23] F. Chapeau-Blondeau, D. Rousseau, Noise-aided SNR amplification by parallel arrays of sensors with saturation, Phys. Lett. A 351 (2006) 231-237.

[24] M.D. McDonnell, N.G. Stocks, C.E.M. Pearce, D. Abbott, Optimal information transmission in nonlinear arrays through suprathreshold stochastic resonance, Phys. Lett. A 352 (2006) 183-189.

[25] F. Duan, F. Chapeau-Blondeau, D. Abbott, Theory of array stochastic resonance in a parallel array of nonlinear dynamical elements, Phys. Lett. A 382 (2008) 2159-2166 
[26] A.R. Bulsara, A. Zador, Threshold detection of wideband signals: a noisecontrolled maximum in the mutual information, Phys. Rev. E 54 (1996) R2185-R2188.

[27] F. Chapeau-Blondeau, Noise-enhanced capacity via stochastic resonance in an asymmetric binary channel, Phys. Rev. E 55 (1997) 2016-2019.

[28] M.E. Inchiosa, J.W.C. Robinson, A.R. Bulsara, Information-theoretic stochastic resonance in noise-floor limited systems: the case of adding noise, Phys. Rev. Lett. 85 (2000) 3369-3372.

[29] L.B. Kish, G.P. Harmer, D. Abbott, Information transfer rate of neurons: stochastic resonance of Shannon's information channel capacity, Fluct. Noise Lett. 1 (2001) L13-L19.

[30] F. Chapeau-Blondeau, A. Delahaies, D. Rousseau, Tsallis entropy measure of noise-aided information transmission in a binary channel, Phys. Lett. A 375 (2011) 2211-2219.

[31] R. Löfstedt, S.N. Coppersmith, Quantum stochastic resonance, Phys. Rev. Lett. 72 (1994) 1947-1950.

[32] I. Goychuk, P. Hänggi, Quantum stochastic resonance in symmetric systems, Phys. Rev. E 59 (1999) 5137-5141.

[33] H.H. Adamyan, S.B. Manvelyan, G.Y. Kryuchkyan, Stochastic resonance in quantum trajectories for an anharmonic oscillator, Phys. Rev. A 63 (2001) 022102.

[34] P.K. Ghosh, D. Barik, D.S. Ray, Noise-induced transition in a quantum system, Phys. Lett. A 342 (2005) 12-21.

[35] J.J.L. Ting, Stochastic resonance for quantum channels, Phys. Rev. E 59 (1999) 2801-2803.

[36] J.J.L. Ting, Noise effects for the depolarizing channel, Phys. Lett. A 259 (1999) 349-354.

[37] J.J.L. Ting, Noise effects on one-Pauli channel, Eur. Phys. J. B 13 (2000) 527-530.

[38] G. Bowen, S. Mancini, Noise enhancing the classical information capacity of a quantum channel, Phys. Lett. A 321 (2004) 1-5.

[39] G. Bowen, S. Mancini, Stochastic resonance effects in quantum channels, Phys. Lett. A 352 (2006) 272-275.

[40] C.W. Helstrom, Quantum detection and estimation theory, J. Stat. Phys. 1 (1969) $231-252$.

[41] C.W. Helstrom, Quantum Detection and Estimation Theory, Academic Press, New York, 1976.

[42] M. Osaki, M. Ban, O. Hirota, Derivation and physical interpretation of the optimum detection operators for coherent-state signals, Phys. Rev. A 54 (1996) 1691-1701.

[43] Y.C. Eldar, G.D. Forney, On quantum detection and the square-root measurement, IEEE Trans. Inf. Theory 47 (2001) 858-872.
[44] R.B.M. Clarke, V.M. Kendon, A. Chefles, S.M. Barnett, E. Riis, M. Sasaki, Experimental realization of optimal detection strategies for overcomplete states, Phys. Rev. A 64 (2001) 012303.

[45] Y.C. Eldar, Von Neumann measurement is optimal for detecting linearly independent mixed quantum states, Phys. Rev. A 68 (2003) 052303.

[46] Y.C. Eldar, A. Megretski, G.C. Verghese, Optimal detection of symmetric mixed quantum states, IEEE Trans. Inf. Theory 50 (2004) 1198-1207.

[47] O. Oreshkov, J. Calsamiglia, R. Muñoz Tapia, E. Bagan, Optimal signal states for quantum detectors, New J. Phys. 13 (2011) 073032

[48] K. Nakahira, K. Kato, T.S. Usuda, Minimax strategy in quantum signal detection with inconclusive results, Phys. Rev. A 88 (2013) 032314.

[49] S.M. Kay, Fundamentals of Statistical Signal Processing: Detection Theory, Prentice Hall, Englewood Cliffs, 1998.

[50] A. Chefles, Unambiguous discrimination between linearly independent quantum states, Phys. Lett. A 239 (1998) 339-347.

[51] T. Rudolph, R.W. Spekkens, P.S. Turner, Unambiguous discrimination of mixed states, Phys. Rev. A 68 (2003) 010301(R).

[52] C. Zhang, Y. Feng, M. Ying, Unambiguous discrimination of mixed quantum states, Phys. Lett. A 353 (2006) 300-306.

[53] U. Herzog, J.A. Bergou, Distinguishing mixed quantum states: minimum-erro discrimination versus optimum unambiguous discrimination, Phys. Rev. A 70 (2004) 022302.

[54] M.E. Deconinck, B.M. Terhal, Qubit state discrimination, Phys. Rev. A 81 (2010) 062304.

[55] K. Hunter, Measurement does not always aid state discrimination, Phys. Rev. A 68 (2003) 012306.

[56] C.W. Gardiner, P. Zoller, Quantum Noise, Springer, Berlin, 2004

[57] S. Haroche, J.-M. Raimond, Exploring the Quantum: Atoms, Cavities, and Photons, Oxford University Press, Oxford, 2006.

[58] A.A. Clerk, M.H. Devoret, S.M. Girvin, F. Marquardt, R.J. Schoelkopf, Introduction to quantum noise, measurement, and amplification, Rev. Mod. Phys. 82 (2010) 1155-1208.

[59] C.M. Caves, J. Combes, Z. Jiang, S. Pandey, Quantum limits on phase-preserving linear amplifiers, Phys. Rev. A 86 (2012) 063802.

[60] M.M. Wilde, Can classical noise enhance quantum transmission?, J. Phys. A 42 (2009) 325301

[61] M.M. Wilde, B. Kosko, Quantum forbidden-interval theorems for stochastic resonance, J. Phys. A 42 (2009) 465309.

[62] C.K. Lee, L.C. Kwek, J. Cao, Stochastic resonance of quantum discord, Phys. Rev. A 84 (2011) 062113

[63] C. Lupo, S. Mancini, M.M. Wilde, Stochastic resonance in Gaussian quantum channels, J. Phys. A 46 (2013) 045306. 